\title{
The Impact of Context on EEG Motor Imagery Neurofeedback and Related Motor Domains
}

\author{
Mareike Daeglau ${ }^{1} \cdot$ Catharina Zich $^{2,3} \cdot$ Cornelia Kranczioch $^{1,4}(\mathbb{0}$
}

Accepted: 7 July 2021/ Published online: 20 August 2021

(c) The Author(s) 2021

\begin{abstract}
Neurofeedback (NF) is a versatile non-invasive neuromodulation technique. In combination with motor imagery (MI), NF has considerable potential for enhancing motor performance or supplementing motor rehabilitation. However, not all users achieve reliable NF control. While research has focused on various brain signal properties and the optimisation of signal processing to solve this issue, the impact of context, i.e. the conditions in which NF motor tasks occur, is comparatively unknown. We review current research on the impact of context on MI NF and related motor domains. We identify long-term factors that act at the level of the individual or of the intervention, and short-term factors, with levels before/after and during a session. The reviewed literature indicates that context plays a significant role. We propose considering context factors as well as within-level and across-level interactions when studying MI NF.
\end{abstract}

\section{Introduction}

Learning new motor skills, improving existing motor skills and re-learning weakened or temporarily lost motor skills requires repeated practice. This practice can have many facets, spanning the whole spectrum from predominantly physical processes to purely mental processes. Some of the most prominent variants include (attempted) motor execution (ME), observation, mirror therapy and motor imagery (MI). The effectiveness of each of these variants in sports performance and rehabilitation is largely uncontroversial. It has been critically noted, however, that variants that fall into the mental end of the spectrum miss feedback on performance,

This article is part of the Topical Collection on Neuromodulation

Cornelia Kranczioch

cornelia.kranczioch@uni-oldenburg.de

1 Neurocognition and Functional Neurorehabilitation Group, Neuropsychology Lab, Department of Psychology, School of Medicine and Health Sciences, Carl Von Ossietzky University Oldenburg, 26111 Oldenburg, Germany

2 Department of Clinical and Movement Neuroscience, UCL Queen Square Institute of Neurology, London, UK

3 Wellcome Centre for Integrative Neuroimaging, FMRIB, Nuffield Department of Clinical Neurosciences, University of Oxford, Oxford, UK

4 Research Center Neurosensory Science, Carl Von Ossietzky University Oldenburg, 26111 Oldenburg, Germany a keystone for directed learning and adaptive cortical reorganisation [1]. A promising solution to this problem is the use of brain-computer-interface (BCI) technology to provide neurofeedback (NF). NF, a closed-loop system, involves measuring an individual's brain activity, analysing the data in real-time and feeding it back to the same individual in an intuitive format. Table 1 provides short explanations of terms and concepts central to MI NF. Like sensory feedback, NF can guide subsequent mental and physical processes. The systematic evaluation of any behavioural gains induced through MI NF practice is generally impeded by the simplicity of applied movements [2-4]. This is because these movements must be suitable for the limited movement capabilities of the clinical target population of paretic stroke patients and must also be appropriate for informative NF. In studies with healthy individuals typically overlearned movements [2-4] can be expected to lead to only small or even not measurable behavioural gains through practice. However, several clinical studies did show behavioural improvements following MI NF practice for these movements (for a review, see [5]). More complex movements may increase the level of difficulty, but may not be transferable to rehabilitation setups, or lack everyday relevance.

Despite numerous technical advances at different points in the NF loop (e.g. online artefact correction, advanced spatial filters, multi-variate classification), which without doubt improve the online signal-to-noise ratio, huge intra- and inter-subject variabilities remain. Understanding the cause(s) 
Table 1 Key terms for motor imagery neurofeedback

Key terms

Motor Imagery

Motor imagery (MI) is defined as mental representation of a particular motor act without observable motor output. Many different forms of MI exist, such as implicit MI and explicit MI. In explicit MI, individuals are asked to imagine a specific movement or action. In implicit MI, individuals are not instructed to imagine a movement but they receive tasks that require the use of MI. A further distinction is made based on the nature of the mental representation. For kinaesthetic MI, individuals are asked to imagine the feeling of a particular motor act, while visual MI usually targets eliciting a 'mental video'. MI can be performed from the first- and third-person perspectives. MI NF focuses on explicit, kinaesthetic MI from the first-person perspective, as this combination is characterised by a greater enrolment of the sensorimotor network and holds thus greater potential for motor rehabilitation

Neuromodulator/neuromodulation

Neuromodulators alter brain activity. Mostly used when an external source is the cause of the modulation, such as brain stimulation, neurofeedback or pharmacological interventions

Neurofeedback

Neurofeedback (NF) and brain-computer-interface (BCI) enable both a direct connection between the brain and an external device. Moreover, both consist of three modules: data acquisition and data processing, the interface and the user. Despite these similarities and the fact that NF and BCI are often used interchangeably, they depict slightly different concepts. In line with the original definitions dating back several decades, NF is to be user-centred, while BCI is application-centred. Consequently, a system with the aim of changing abnormal brain activation patterns is classed as NF, whereby a system with the aim of steering a device (e.g. a cursor or a wheelchair) is classed as BCI SMR

Sensorimotor rhythm (SMR) refers to activity in the mu $(8-12 \mathrm{~Hz})$ and beta $(13-30 \mathrm{~Hz})$ frequency range stemming from sensorimotor areas. During cognitive-motor tasks and movement execution SMR activity typically decreases before and during the act, known as event-related desynchronisation (ERD), and increases afterward, known as event-related synchronisation (ERS). Most MI NFs are based on SMR ERD. Motor learning has been associated with changes in both ERD and ERS

underlying these variabilities is key to further improve NF success and thus the learning, improving and re-learning of motor skills. Technical factors that could account for these variabilities have been investigated in depth. Here, we focus on what we call context factors. The Webster-Meriam dictionary defines context as 'the interrelated conditions in which something exists or occurs' (https://www.merriamwebster.com/dictionary/context). Borrowing from this basic definition, we define context as the interrelated conditions in which (NF) motor tasks occur. Context factors can be relatively stable and long-term in that they cannot or would normally not change for an intervention period. Long-term context factors can be subdivided into factors regarding the individual (e.g. age, gender, personality or capability to perform the movement) and factors regarding the intervention (e.g. social context, environment, NF implementation, see Fig. 1). Context factors can also be short-term in that they can change from session to session (e.g. time of intervention in relation to time of sleep, circadian rhythm, nutrition, physical exercise or interference tasks) or even within a session (e.g. state motivation, attention and stress, experimenter gender). In this narrative review, we will give a brief overview of recent literature on such context factors. We will put special emphasis on MI NF, with the NF based on spectral neural signals measured with the electroencephalogram (EEG, see Table 2 for a summary of references from 2017 onward). Yet, despite the focus on MI NF, most context factors will be equally relevant for related domains.

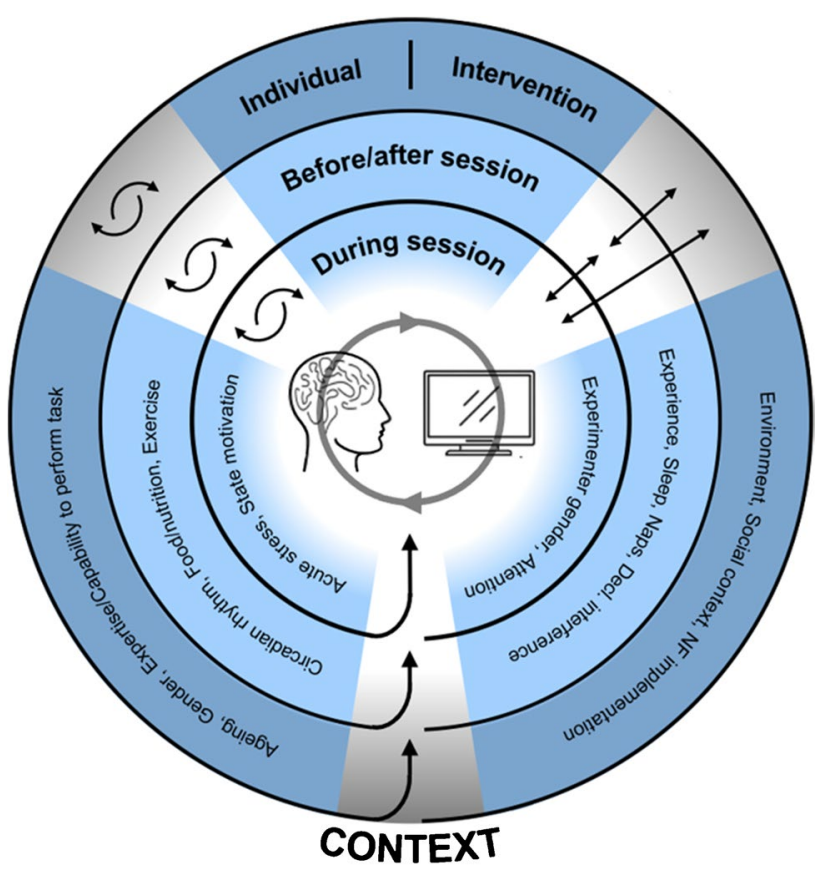

Fig. 1 Levels of context factors and their interactions. Differentiated is between long-term factors (dark blue) that act at the level of the individual or of the intervention, and short-term factors (light blue), with levels before/after and during a session. Factors can interact within levels (arrows top left) and between levels (arrows top right). Examples for interactions could be 'Experimenter gender' and 'Acute stress' within level, and 'Declarative interference' and 'Acute stress' between level. MI NF is under the influence of both, individual factors, and the consequences of within- and between-level interactions (bottom arrows) 


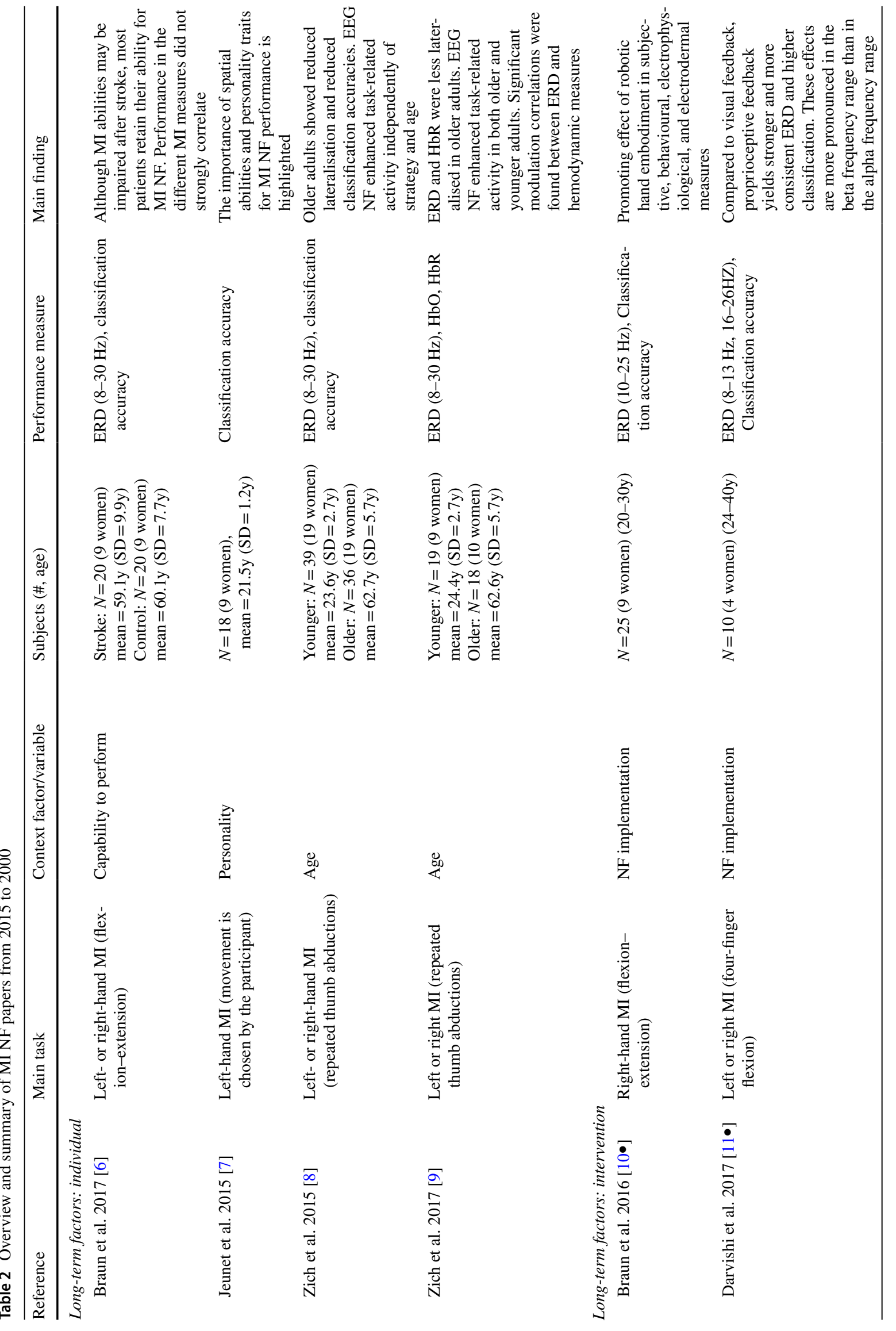




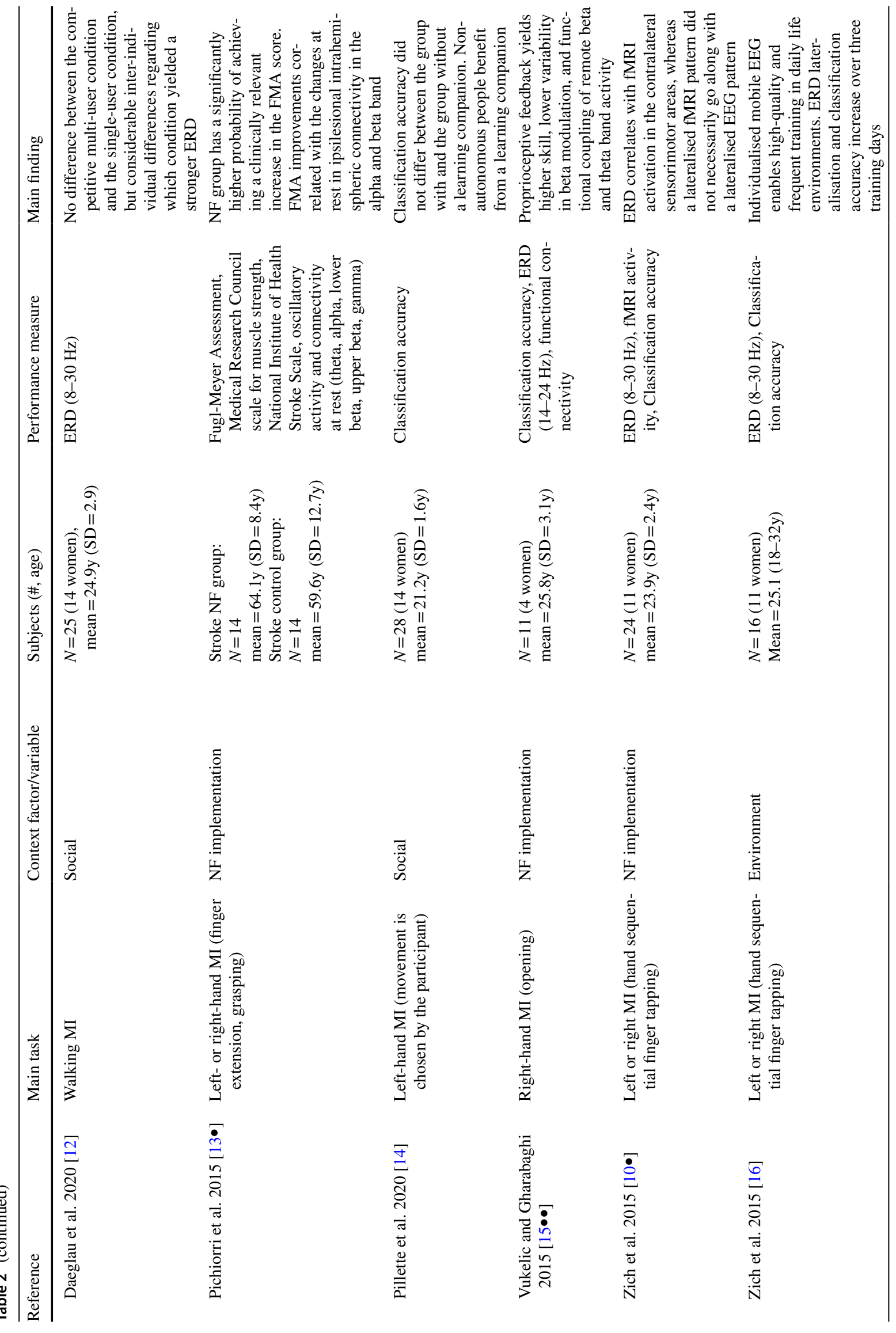




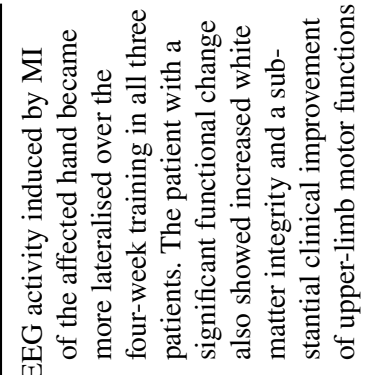

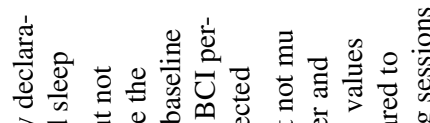

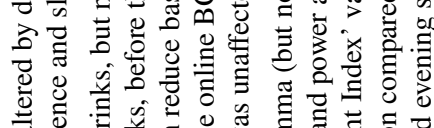

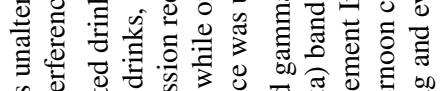

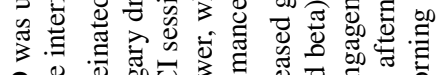

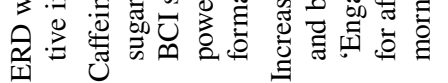

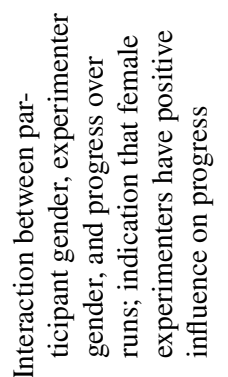

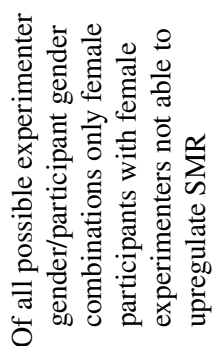

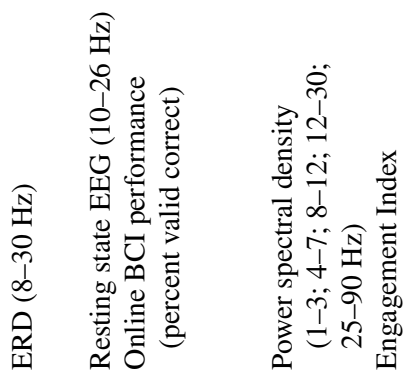

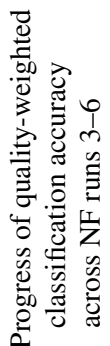

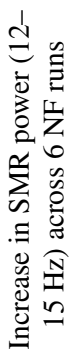

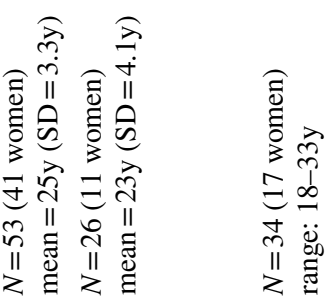

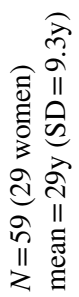

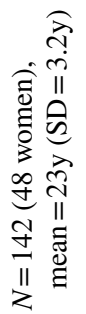

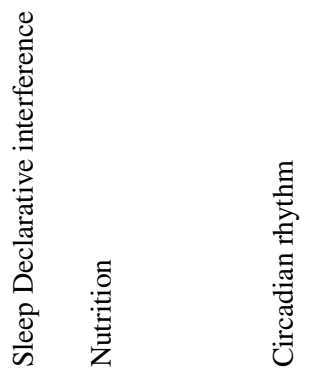

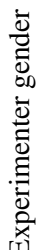

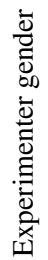
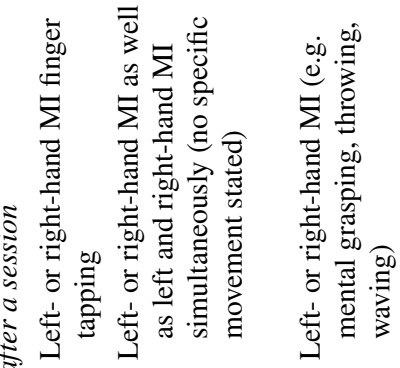

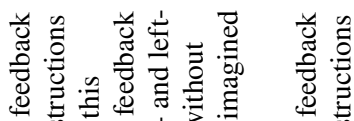

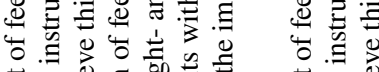
공늘 ब0 की

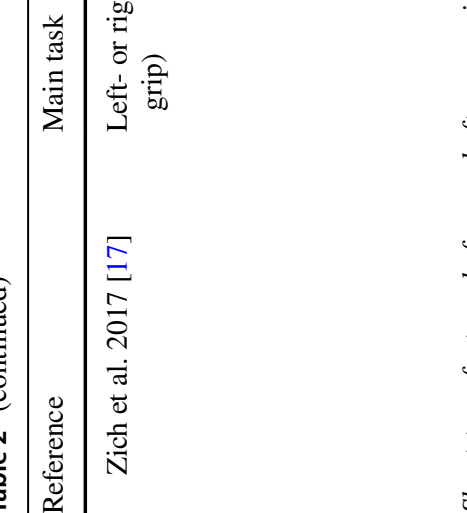

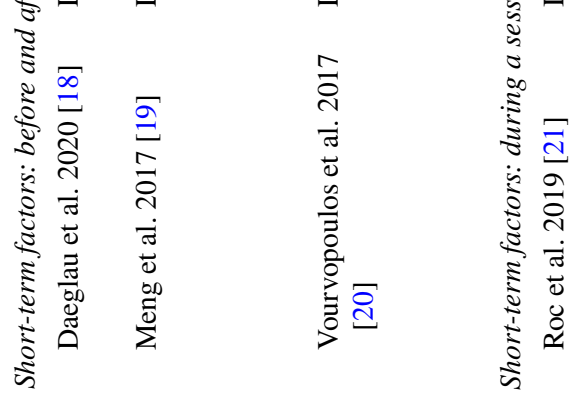

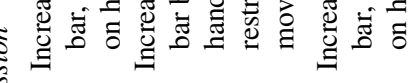




\section{Long-term Factors: Individual}

Normal aging is accompanied by anatomical and functional changes and MI NF performance is no exception to this. Older adults perform MI as vividly as younger adults [23-25]; however, MI quality [24] and accuracy of estimating temporal and spatial characteristics of MI are altered (explicit MI: [8, 9], implicit MI: [26]). These age-related behavioural changes are accompanied by neurofunctional changes, i.e. more widespread and symmetric activity in older adults [27], which are in line with the HAROLD model [28]. Similar results could be observed for MI NF [29, 30]. Moreover, when the neural feature which serves as the basis for the NF is subject to age-related changes the MI NF performance is likely altered as well [29].

Regarding gender, while men show better target abilities in gross movements [31], women are better in fine movements [32]. MI NF comprises mostly small hand movements, which is why women might have an advantage. However, MI NF comprises additional mental and technical elements that can have an effect. Regarding the mental element, for MI, no gender difference has been observed [7, 25]. However, regarding the technical element, it has been found that control beliefs while dealing with technology [33], which are generally higher in men [33], correlate negatively with MI NF performance ([34], but see also [35]). Witte and colleagues interpret their findings as follows: individuals with strong control beliefs try harder to control the NF and thus activate potentially interfering resources. This is in line with a finding on personality traits indicating a negative relationship between MI NF performance and tension [36]. The same study reported that MI NF performance was positively related to self-reliance and abstractness. However, the correlation between personality traits and MI NF performance was too small to justify the inclusion of personality traits in the prediction model for MI NF performance [37].

Expertise and capability to perform the to-be-imagined movement constitute further context factors. Compared to experts, novices recruit additional resources during MI ([38], for reviews, see [6, 39]), which has been linked to increased cognitive demand [40]. In healthy individuals, short-term limb immobilisation led to selective impairment of MI ability [41, 42]. Post-stroke some studies found MI, in general, to be altered [43, 44•], while others found specific aspects of MI to be altered [12, 14] or no alterations [16, 17]. Interestingly, Braun et al. [44•] found that although MI abilities may be impaired after stroke, most patients retain their ability for MI NF.

\section{Long-term Factors: Intervention}

The number of individuals participating in the same session constitutes its social context. Although practicing in a group has several advantages, MI NF is traditionally conducted alone and only a few studies investigated the effect of social context on MI NF. Initial reports suggest that, compared to a single-user and a competitive multi-user condition, a collaborative multi-user condition is generally more enjoyable and motivating [45]. However, inter-individual differences exist concerning which social context yields stronger neural activity during MI NF [10•]. Considerations on the importance of social context have inspired the development of a personalised emotional agent for NF [11•].

MI NF is primarily conducted in laboratory-based environments. While this instigates methodological rigour, MI NF at home enables, regarding the context factor environment, familiar surroundings and no need to travel. These are key elements of the so-called home advantage, which has a positive effect in sport and probably also for MI NF. While technical aspects of laboratory- and home-based settings have been compared [3] the impact of the context factor environment on MI NF success has not been formally studied yet. Despite this gap, MI NF at home gains popularity [15••], probably also because it is less cost-intensive, more inclusive and enables more frequent and more ecologically valid training [46].

While some aspects of the NF will be adapted on a sessionto-session basis (e.g. spatial and temporal properties of the extracted EEG signals, classifiers for the NF), the overall $N F$ implementation is likely to be constant throughout the training. While MI NF can be based on nearly any recording technique or combination of techniques (so-called hybrid approaches, e.g. EEG-fNIRS, EEG-fMRI), EEG-based NF is most common. Crucially, for EEG it has been shown that not every individual will show the neural feature which serves as the basis for the NF. These cases would likely benefit from hybrid implementations or a complete switch of recording technique [47]. Regarding the feedback modality, proprioceptive NF yields stronger and less variable task-related modulation in power than visual NF $[13 \bullet, 48]$. This is in line with the notion that realistic and embodiable NF (robotic hand: [49]; virtual environment: [50]; electrical stimulation: [51]) is advantageous. For both, visual and proprioceptive NFs, it has been shown that positive feedback is preferable $[52,53]$.

\section{Short-term Factors: Before and After a Session}

It has been suggested that experience with a given movement is necessary for correct and vivid MI [54-56]. Yet, often, novel movements with which participants have only limited experience are used (for a review, see [57]). In a recent study, we aimed to evaluate the effect of extended ME practice in a visuo-motor task. We did not find evidence that ME practice leads to a stronger subsequent MI-induced ERD of the same movement in comparison to no prior ME practice [58]. This contrasts with previous studies reporting priming 
effects (ME on MI: [56]; MI on ME: [57, 58]), which we currently interpret, as an indication that already rather small differences between setups can lead to deviant or opposing findings. To only name two aspects which may have prevented measurable behavioural and neural gains following MI [58] in our study, we did not include a preparation phase before the beginning of each trial, and the motor task was slightly more complex than in other studies. Further research is necessary to shed light on the specific relevance of these and other factors for MI NF.

Sleep is a key component for the offline phase (i.e. after practice: $[59,60])$ of motor learning, improving and relearning (for a review, see [61]). Several studies on motor skill acquisition have shown beneficial effects of sleep following motor skill practice on subsequent motor performance (for an extensive review, see [62]). However, this well-established finding was challenged lately, for instance by evidence that supposedly sleep-related performance gains are not exceeding performance levels already achieved shortly after a practice session, e.g. [63-66]. This 'early boost' may represent an offline performance gain, and can be measured around 5-30 min after motor (imagery) practice and then declines over the next 4-12 h of wakefulness [18, 67, 68]. More recent studies indicate differences regarding the practice modality, that is, MI or ME and task complexity for gains following a night of sleep. Freitas and colleagues found no offline gain in ME performance after combining ME practice and sleep, but when MI practice was followed by sleep, ME performance further improved compared to performance right after practice [69]. MI practice resulted in an additional performance increase after sleep compared to consolidation over the day but only for a complex movement sequence [70]. The finding that the effects were restricted to the complex movement is partially in line with recent findings on MI NF performance on a simple MI task that neither indicate the presence of an early boost nor of sleep-related performance gains [71]. Interestingly, in contrast to the relatively rich body of research on sleep, the impact of sleep deprivation on motor performance remains unclear [72].

In addition to the effects of a full night of sleep, daytime naps have also been investigated lately. For ME practice, Backhaus and colleagues found that short daytime naps did not have a positive impact on offline learning and sleepdependent consolidation for both explicit motor sequence and motor adaptation practice tasks in older adults, but rather led to performance deterioration [73]. In contrast, a daytime nap after MI practice was found to improve motor performance [74]. Debarnot and colleagues [75] found adverse effects of subsequent declarative interference, that is, of performing tasks drawing on declarative memory, on the positive effects of MI practice on ME, both over intervals of sleep and wakefulness. Adverse effects have been reported to be less sustainable for ME practice, where motor consolidation is impaired by declarative interference over wakefulness but recovers over a period of sleep [76]. However, no evidence for any adverse effect of various verbal and non-verbal declarative interference tasks on $\mathrm{ME}$ task performance was found in a more recent study [77]. In line with the latter, we found no evidence for any impact of declarative interference on MI NF performance, neither over wakefulness nor after a night of sleep [71].

Circadian rhythm and chronotype have been studied for ME, MI and MI NF. Scheduling practice sessions according to athlete's circadian preferences yielded significantly better ME performance [78]. Also, learning new movement sequences in the morning induces an increase in corticomotor excitability in the primary motor cortex that is absent when learning new sequences in the evening ([79], but see [80]). For MI time (i.e. time to imagine a given task) and MI chronometry (i.e. temporal congruency between ME and MI), Debarnot and colleagues found modulations based on circadian rhythm, but rather unsystematic and task-dependent [20]. This finding was supported [81] and extended to the factor motor imagery quality [82]. In an MI BCI gaming paradigm significantly increased gamma, but not mu and beta, band power and increased 'Engagement Index' values have been reported for afternoon compared to morning and evening sessions [83].

Insights on the role of nutrition/food intake on motor performance come, for instance, from fasting: while two days of strict fasting in obese women had no effects on handgrip strengths and psychomotor coordination [19], a period of Ramadan fasting reduced agility, speed and reaction time performance for male tennis players [84]. This is in line with a recent meta-analysis showing that dehydration impairs performance in tasks involving attention, executive function and motor coordination when water deficits exceed $2 \%$ body mass loss [85]. Moreover, while caffeinated drinks, but not sugary drinks, before the BCI session were found to reduce baseline power in the mu and beta frequency range, online BCI performance was unaltered [86].

Physical exercise unrelated to the motor task under investigation expedites motor learning, improvement, and re-learning and learning-related neural activity [87, 88]. This also holds for the MI SMR within the mu frequency range [22]. Furthermore, a progressive muscular relaxation (PMR) intervention before MI NF was found to boost NF performance [21].

\section{Short-term Factors: During a Session}

Across a wide range of domains, it has been shown that in experiments with humans, experimenter gender can have an influence on experimental findings [89]. Little 
surprisingly, this also holds for MI NF. Wood and Kober [90•] trained male and female participants by either male or female experimenters and found that female participants trained by female experimenters did not show a gain in the NF signal. Another study however indicated an overall positive effect of female experimenters on MI NF performance in male participants, and mixed effects of experimenter gender on performance in female participants [91]. Thus, though in general, women seem to perform better with male experimenters and vice versa [89], for MI NF performance, the direction of the effect appears less clear. A factor contributing to the latter could be how the purpose of the NF session is-inadvertent or intended-perceived by the participant, i.e. as technologic or therapeutic [90•]. The generally seen opposite-sex performance benefit has been attributed to an aggregation of moderate levels of psychosocial stress and the heightened reward potential of opposite-sex interactions [92]. The findings by Wood and Kober [90•] and Roc et al. [91] are both partially in line with this. Recent evidence from the motor learning domain underline however that also detrimental oppositesex effects can occur [92, 93], indicating that in some setups, psychosocial stress levels induced by opposite-sex experimenters can become so high that performance drops. Acute stress, experimentally induced through pain and social evaluation, has been found to reduce performance in implicit but not in explicit MI [94]. As MI NF relies on explicit MI, it can be expected that it is also sensitive to acute stress, though this has not been tested yet. For motor learning, effects of acute stress have also been reported, but with diverging outcomes. One recent study, inducing stress through pain and social evaluation, found no effect of acute stress on initial motor performance, training gain and motor memory consolidation at the group level. The acute stress group data indicated however a negative correlation between overnight gains in performance and the stress-induced cortisol level [95]. This contrasts with a study in which stress and anxiety were induced through a computerised mental arithmetic task and whose results indicate positive effects of acute stress on training gain and retention [96].

Adding NF to MI can raise or keep up motivation, and, as discussed above, it is assumed that this depends, among others, on the NF implementation. The motivation added by the NF is met by the general motivation of a participant to participate. Though the motivation to participate in a MI NF study is most likely derived from several sources, for the prototypical healthy participant, it will be predominantly extrinsically driven, with money or course credits as main rewards. For patients, such external rewards will be of comparatively little importance. Their motivation will be much more intrinsically driven, for instance by the wish to learn about new therapeutic options or by the hope to improve function. Yet even in participants with a strong intrinsic motivation to participate such as chronic stroke patients, state motivation, that is, the motivation for a given session, varies between sessions $[15 \bullet \bullet$. Several older studies with healthy participants indicate that state motivation can have an influence on MI NF performance [97-99]. In these studies, state motivation was operationalised along several dimensions. The dimensions 'perceived challenge' and 'interest' correlated positively with performance. For the dimension 'fear of incompetence', overall, a negative relationship was found [97-99]. A longitudinal study with patients suffering from amyotrophic lateral sclerosis (ALS) also reported a positive correlation between 'perceived challenge' and performance, but only for one of six patients and no significant correlation for the remaining five patients [100]. This and related findings for stroke patients [97] suggest that for patients, the link between state motivation and MI NF performance might differ from healthy participants. We found no recent literature following up on these earlier reports on motivation though, corroborating this observation. Yet irrespective of this, it is a fact that to some degree MI NF training will have to put up with fluctuations in motivation, as reasons can be personal and entirely unrelated to the NF training in itself. At the same time, experimenters or therapists should also be open to the possibility that their conduct can have an influence on state motivation and thus, potentially, on NF performance. This is suggested by the finding that a motor learning setup in which the experimenter supports the human psychological need of relatedness [101] by emphasising caring and interest in the participant's experiences improves not only motivation and positive affect but also motor learning and retention [102].

The aspects discussed so far can change between sessions but are probably rather stable within a session. Other aspects, such as the ability to focus on the task or attention, are likely to change on a shorter time scale, that is, from trial to trial or as a function of time within a session. To learn more about these fluctuations and how they affect MI NF or BCI performance, the period before the start of the actual MI task has been mined for information. While an early study successfully predicted trial-by-trial performance of an SMR-based MI NF with fluctuations in gamma activity [103], subsequent studies derived predictors from various combinations of at least three frequency bands, including the delta, theta, alpha, beta and gamma bands [104, 105]. Importantly, the best-performing combination of predictors was found to differ between participants [105]. The individuality of best-performing predictors is in line with findings on predicting ME performance based on spectral pre-trial EEG activity [106]. 


\section{Prospects and Concluding Remarks}

In this narrative review, we highlight a range of long- and short-term context factors summarised in Fig. 1 that can influence the learning, improvement and re-learning of motor skills. We primarily focused on MI NF, whereby this was supplemented by literature from related domains, such as MI without NF and ME. Nevertheless, this review is not exhaustive and other context factors will certainly also play their role. One such factor constitutes other additional neuromodulation techniques, such as pharmacological interventions (e.g. GABAB receptor agonist on ME learning [107] or brain stimulation e.g. transcranial direct current stimulation (tDCS) and MI NF: [108-111] or transcranial alternating current stimulation (tACS) and MI NF: [112]), if applied before/after or during the session.

For several of the context factors reviewed, contradictory results were found. One important aspect of this may be the nature of the motor task. A wealth of motor tasks is used in research, and, in other frameworks, task variability served as an explanatory variable for variability in results, both across individuals and studies e.g. [20, 70, 73]. On the other hand, it is also conceivable that the effect of context factors is largely independent of the motor task and that the differences in findings have another origin, such as the interaction between context factors or individual preferences (cf. section social context) and/or individual physiological differences $[113,114]$. Future research will have to investigate the merits of this case.

With this first review on context factors in MI NF and related domains, we focus on the main effects, i.e. the independent and direct influence of each factor. Beyond that, however, interactions can occur within each level, e.g. between two or more long-term factors, and, across levels, e.g. factors that are relevant before the session can interact with factors that are relevant during the session. We believe that from considering main effects and interactions our understanding of the impact of context factors on MI NF performance and related domains will greatly benefit.

Funding Open Access funding enabled and organized by Projekt DEAL.

Open Access This article is licensed under a Creative Commons Attribution 4.0 International License, which permits use, sharing, adaptation, distribution and reproduction in any medium or format, as long as you give appropriate credit to the original author(s) and the source, provide a link to the Creative Commons licence, and indicate if changes were made. The images or other third party material in this article are included in the article's Creative Commons licence, unless indicated otherwise in a credit line to the material. If material is not included in the article's Creative Commons licence and your intended use is not permitted by statutory regulation or exceeds the permitted use, you will need to obtain permission directly from the copyright holder. To view a copy of this licence, visit http://creativecommons.org/licenses/by/4.0/.

\section{References}

Papers of particular interest, published recently, have been highlighted as:

- Of importance

$\bullet$ Of major importance

1. Langhorne P, Coupar F, Pollock A. Motor recovery after stroke: a systematic review. Lancet Neurol. 2009;8(8):741-54.

2. Nikulin VV, Hohlefeld FU, Jacobs AM, Curio G. Quasi-movements: a novel motor-cognitive phenomenon. Neuropsychologia. 2008;46(2):727-42.

3. Zich C, De Vos M, Kranczioch C, Debener S. Wireless EEG with individualized channel layout enables efficient motor imagery training. Clin Neurophysiol. 2015;126(4):698-710.

4. Grosprêtre S, Ruffino C, Lebon F. Motor imagery and corticospinal excitability: a review. Eur J Sport Sci. 2016;16(3):317-24.

5. Mane R, Chouhan T, Guan C. BCI for stroke rehabilitation: motor and beyond J Neural Eng. 2020; 17(4). https://doi.org/10. 1088/1741-2552/aba162.

6. Yang J. The influence of motor expertise on the brain activity of motor task performance: a meta-analysis of functional magnetic resonance imaging studies. Cogn Affect. Behav Neurosci. 2015. https://doi.org/10.3758/s13415-014-0329-0.

7. Campos A. Gender differences in imagery. Pers Individ Dif. 2014;59:107-11.

8. Gabbard C, Caçola P, Cordova A. Is there an advanced aging effect on the ability to mentally represent action? Arch Gerontol Geriatr. 2011;53(2):206-9.

9. Personnier P, Kubicki A, Laroche D, Papaxanthis C. Temporal features of imagined locomotion in normal aging. Neurosci Lett. 2010;476(3):146-9.

10. Daeglau M, Wallhoff F, Debener S, Condro IS, Kranczioch C, Zich C. Challenge accepted? Individual performance gains for motor imagery practice with humanoid robotic EEG neurofeedback. Sensors (Switzerland). 2020; 20(6). https://doi.org/10. 3758/s13415-014-0329-0. Find no difference between a competitive multi-user condition and a single-user condition, but considerable inter-individual differences regarding which condition yielded a stronger ERD.

11.• Pillette L, Jeunet C, Mansencal B, N'Kambou R, N'Kaoua B, Lotte F. A physical learning companion for mental-imagery BCI user training. Int J Hum Comput Stud. 2020. https://doi.org/10. 1016/j.ijhcs.2019.102380. Introduce a humanoid learning companion but see no difference in classification accuracy between the group with and the group without the companion. However, non-autonomous people benefit from the learning companion.

12. Liepert J, Büsching I, Sehle A, Schoenfeld MA. Mental chronometry and mental rotation abilities in stroke patients with different degrees of sensory deficit. Restor Neurol Neurosci. 2016;34(6):907-14.

13. Darvishi S, Gharabaghi A, Boulay CB, Ridding MC, Abbott D, Baumert M. Proprioceptive feedback facilitates motor imageryrelated operant learning of sensorimotor $\beta$-band modulation. Front Neurosci. 2017;11(FEB):1-13. Comparison of visual and proprioceptive feedback. Proprioceptive feedback yielded stronger and more consistent ERD and higher classification, effects more pronounced in the beta frequency range than in the alpha frequency range.

14. de Vries S, Tepper M, Feenstra W, Oosterveld H, Boonstra AM, Otten B. Motor imagery ability in stroke patients: the relationship between implicit and explicit motor imagery measures. 
Front Hum Neurosci. 2013. https://doi.org/10.3389/fnhum.2013. 00790.

15.••Zich C, Debener S, Schweinitz C, Sterr A, Meekes J, Kranczioch C. High-intensity chronic stroke motor imagery neurofeedback training at home: three case reports. Clin EEG Neurosci. 2017;48(6):403-12. https://doi.org/10.1177/1550059417 717398. MI NF training with small and mobile EEG setup. EEG activity was more lateralised over the 4-week training in all three patients, increased white matter integrity, and a substantial clinical improvement of upper-limb motor functions in one.

16. Johnson SH, Sprehn G, Saykin AJ. Intact motor imagery in chronic upper limb hemiplegics: evidence for activityindependent action representations. J Cogn Neurosci. 2002;14(6):841-52.

17. Johnson SH. Imagining the impossible: intact motor representations in hemiplegics. NeuroReport. 2000;11(4):729-32.

18. Hotermans C, Peigneux P, De Noordhout AM, Moonen G, Maquet P. Early boost and slow consolidation in motor skill learning. Learn Mem. 2006;13(5):580-3.

19. Solianik R, Sujeta A. Two-day fasting evokes stress, but does not affect mood, brain activity, cognitive, psychomotor, and motor performance in overweight women. Behav Brain Res. 2018;338:166-72.

20. Debarnot U, Sahraoui D, Champely S, Collet C, Guillot A. Selective influence of circadian modulation and task characteristics on motor imagery time. Res Q Exerc Sport. 2012. https:// doi.org/10.5641/027013612802573111.

21. Acqualagna L, Botrel L, Vidaurre C, Kübler A, Blankertz B. Large-scale assessment of a fully automatic co-adaptive motor imagery-based brain computer interface. PLoS One. 2016; 11(2). https://doi.org/10.1371/journal.pone.0148886.

22. Wriessnegger SC, Brunner C, Müller-Putz GR. Frequency specific cortical dynamics during motor imagery are influenced by prior physical activity Front Psychol. 2018; 9(OCT). https://doi. org/10.3389/fpsyg.2018.01976.

23. Saimpont A, Malouin F, Tousignant B, Jackson PL. Assessing motor imagery ability in younger and older adults by combining measures of vividness, controllability and timing of motor imagery. Brain Res. 2015. https://doi.org/10.1016/j.brainres. 2014.11.050.

24. Malouin F, Richards CL, Durand A. Normal aging and motor imagery vividness: implications for mental practice training in rehabilitation. Arch Phys Med Rehabil. 2010;91(7):1122-7.

25. Subirats L, Allali G, Briansoulet M, Salle JY, Perrochon A. Age and gender differences in motor imagery. J Neurol Sci. 2018;391(June):114-7.

26. Devlin AL, Wilson PH. Adult age differences in the ability to mentally transform object and body stimuli. Aging Neuropsychol Cogn. 2010;17(6):709-29.

27. Kaiser V, Kreilinger A, Müller-Putz GR, Neuper C. First steps toward a motor imagery based stroke BCI: New strategy to set up a classifier. Front Neurosci. 2011;5(JUL):86.

28. Cabeza R. Hemispheric asymmetry reduction in older adults: the HAROLD modelR. Psychol. Aging. 2002; 17(1). https://doi.org/ 10.1037/0882-7974.17.1.85.

29. Zich C, Debener S, De Vos M, Frerichs S, Maurer S, Kranczioch C. Lateralization patterns of covert but not overt movements change with age: an EEG neurofeedback study. Neuroimage. 2015;116:80-91.

30. Zich C, Debener S, Thoene AK, Chen LC, Kranczioch C. Simultaneous EEG-fNIRS reveals how age and feedback affect motor imagery signatures. Neurobiol Aging. 2017;49:183-97.

31. Moreno-Briseño P, Díaz R, Campos-Romo A, Fernandez-Ruiz J. Sex-related differences in motor learning and performance. Behav Brain Funct. 2010;6(1):74.
32. Liutsko L, Muiños R, Ral JMT, Contreras MJ. Fine motor precision tasks: sex differences in performance with and without visual guidance across different age groups. Behav. Sci. (Basel). 2020; 10(1). https://doi.org/10.3390/bs 10010036.

33. Beier G. Kontrollüberzeugungen im Umgang mit Technik. Rep. Psychol. 1999; 684-693.

34. Witte M, Kober SE, Ninaus M, Neuper C, Wood G. Control beliefs can predict the ability to up-regulate sensorimotor rhythm during neurofeedback training. Front Hum Neurosci. 2013;7(JUL):478.

35. Burde W, Blankertz B. Is the locus of control of reinforcement a predictor of brain-computer interface performance?, in Proceedings of the 3rd International BrainComputer Interface Workshop and Training Course, 2006; 2006, pp. 108-109.

36. Jeunet C, Nkaoua B, Subramanian S, Hachet M, Lotte F. Predicting mental imagery-based BCI performance from personality, cognitive profile and neurophysiological patterns. PLoS One. 2015;10(12):1-21.

37. Hammer EM, et al. Psychological predictors of SMR-BCI performance. Biol Psychol. 2012. https://doi.org/10.1016/j.neuro image.2010.03.022.

38. Kraeutner SN, McWhinney SR, Solomon JP, Dithurbide L, Boe SG. Experience modulates motor imagery-based brain activity. Eur J Neurosci. 2018;47(10):1221-9.

39. Debarnot U, Sperduti M, Di Rienzo F, Guillot A. Experts bodies, experts minds: how physical and mental training shape the brain. Front Hum Neurosci. 2014. https://doi.org/10.5641/0270136128 02573111.

40. Orlandi A, Arno E, Proverbio AM. The effect of expertise on kinesthetic motor imagery of complex actions. Brain Topogr. 2020. https://doi.org/10.1007/s10548-020-00760-x.

41. Meugnot A, Agbangla NF, Almecija Y, Toussaint L. Motor imagery practice may compensate for the slowdown of sensorimotor processes induced by short-term upper-limb immobilization. Psychol. Res. 2015. https://doi.org/10.1007/ s00426-014-0577-1.

42. Meugnot A, Agbangla NF, Toussaint L. Selective impairment of sensorimotor representations following short-term upper-limb immobilization. Q J Exp Psychol. 2016;69(9):1842-50.

43. Malouin F, Richards CL, Desrosiers J, Doyon J. Bilateral slowing of mentally simulated actions after stroke. NeuroReport. 2004;15(8):1349-53.

44. Braun N, et al. Motor imagery impairment in postacute stroke patients. Neural Plast. 2017;2017:1-13. https://doi.org/10. $1155 / 2017 / 4653256$. Shows that though MI abilities may be impaired after stroke, most patients retain their ability for MI NF. Performance in the different MI measures did not strongly correlate.

45. Bonnet L, Lotte F, Lécuyer A. Two brains, one game: design and evaluation of a multiuser bci video game based on motor imagery. IEEE Trans Comput Intell AI Games. 2013;5(2):185-98.

46. Kranczioch C, Zich C, Schierholz I, Sterr A. Mobile EEG and its potential to promote the theory and application of imagery-based motor rehabilitation. Int J Psychophysiol. 2014;91(1):10-5.

47. Zich C, Debener S, Kranczioch C, Bleichner MG, Gutberlet I, De Vos M. Real-time EEG feedback during simultaneous EEGfMRI identifies the cortical signature of motor imagery. Neuroimage. 2015. https://doi.org/10.1016/j.neuroimage.2015.04.020.

48. Vukelić M, Gharabaghi A. Oscillatory entrainment of the motor cortical network during motor imagery is modulated by the feedback modality. Neuroimage. 2015; 111. https://doi.org/10.1016/j. neuroimage.2015.01.058.

49. Braun N, Emkes R, Thorne JD, Debener S. Embodied neurofeedback with an anthropomorphic robotic hand. Sci Rep. 2016;6(November):1-13. 
50. Ono T, Kimura A, Ushiba J. Daily training with realistic visual feedback improves reproducibility of event-related desynchronisation following hand motor imagery. Clin Neurophysiol. 2013;124(9):1779-86.

51. Pichiorri F, et al. Brain-computer interface boosts motor imagery practice during stroke recovery. Ann Neurol. 2015;77(5):851-65.

52. González-Franco M, Yuan P, Zhang D, Hong B, Gao S. Motor imagery based brain-computer interface: a study of the effect of positive and negative feedback, in Proceedings of the Annual International Conference of the IEEE Engineering in Medicine and Biology Society, EMBS; 2011.

53. Ramos-Murguialday A, et al. Proprioceptive feedback and brain computer interface (BCI) based neuroprostheses. PLoS One. 2012; 7(10). https://doi.org/10.1371/journal.pone.0047048.

54. Jackson PL, Lafleur MF, Malouin F, Richards CL, Doyon J. Functional cerebral reorganization following motor sequence learning through mental practice with motor imagery. Neuroimage. 2003;20(2):1171-80.

55. Mulder T, Zijlstra S, Zijlstra W, Hochstenbach J. The role of motor imagery in learning a totally novel movement. Exp Brain Res. 2004;154(2):211-7.

56. Sobierajewicz J, Szarkiewicz S, Przekoracka-Krawczyk A, Jaskowski W, Van Der Lubbe R. To what extent can motor imagery replace motor execution while learning a fine motor skill? Adv Cogn Psychol. 2016. https://doi.org/10.5709/ acp-0197-1.

57. Hétu S, et al. The neural network of motor imagery: an ALE meta-analysis. Neurosci Biobehav Rev. 2013;37(5):930-49.

58. Daeglau M, Zich C, Emkes R, Welzel J, Debener S, Kranczioch C. Investigating priming effects of physical practice on motor imagery-induced event-related desynchronization. Front Psychol. 2020; 11(February). https://doi.org/10.3389/fpsyg.2020. 00057.

59. Williams SE, Cumming J, Edwards MG. The functional equivalence between movement imagery, observation, and execution influences imagery ability. Res Q Exerc Sport. 2011. https://doi. org/10.1080/02701367.2011.10599788.

60. Allami N, Brovelli A, Hamzaoui EM, Regragui F, Paulignan Y, Boussaoud D. Neurophysiological correlates of visuo-motor learning through mental and physical practice. Neuropsychologia. 2014;55(1):6-14.

61. Di Rienzo F, et al. Online and offline performance gains following motor imagery practice: a comprehensive review of behavioral and neuroimaging studies. Front Hum Neurosci. 2016;10(June):1-15.

62. King BR, Hoedlmoser K, Hirschauer F, Dolfen N, Albouy G. Sleeping on the motor engram: the multifaceted nature of sleeprelated motor memory consolidation. Neurosci Biobehav Rev. 2017;80:1-22.

63. Nettersheim A, Hallschmid M, Born J, Diekelmann S. The role of sleep in motor sequence consolidation: stabilization rather than enhancement. J Neurosci. 2015;35(17):6696-702.

64. Landry S, Anderson C, Conduit R. The effects of sleep, wake activity and time-on-task on offline motor sequence learning. Neurobiol Learn Mem. 2016;127:56-63.

65. Rickard TC, Cai DJ, Rieth CA, Jones J, Ard MC. Sleep Does Not Enhance Motor Sequence Learning. J Exp Psychol Learn Mem Cogn. 2008. https://doi.org/10.1037/0278-7393.34.4.834.

66. Pan SC, Rickard TC. Sleep and motor learning: Is there room for consolidation? Psychol Bull. 2015. https://doi.org/10.1037/ bul0000009.

67. Brawn TP, Fenn KM, Nusbaum HC, Margoliash D. Consolidating the effects of waking and sleep on motor-sequence learning. J Neurosci. 2010;30(42):13977-82.

68. Debarnot U, Clerget E, Olivier E. Role of the primary motor cortex in the early boost in performance following mental imagery training. PLoS One. 2011; 6(10). https://doi.org/10.1371/journ al.pone.0026717.

69. Freitas E, Saimpont A, Blache Y, Debarnot U. Acquisition and consolidation of sequential footstep movements with physical and motor imagery practice. Scand J Med Sci Sport. 2020;30(12):2477-84.

70. Debarnot U, Abichou K, Kalenzaga S, Sperduti M, Piolino P. Variable motor imagery training induces sleep memory consolidation and transfer improvements. Neurobiol Learn Mem. 2015. https://doi.org/10.1016/j.nlm.2014.12.010.

71. Daeglau M, Zich C, Welzel J, Saak SK, Scheffels JF, Kranczioch C. Motor imagery EEG neurofeedback skill acquisition in the context of declarative interference and sleep. bioRxiv. 2020. https://doi.org/10.1101/2020.12.11.420919.

72. Vaara JP, Oksanen H, Kyröläinen H, Virmavirta M, Koski H, Finni T. 60-hour sleep deprivation affects submaximal but not maximal physical performance. Front Physiol. 2018; 9(OCT). https://doi.org/10.3389/fphys.2018.01437.

73. Backhaus W, Braass H, Renné T, Gerloff C, Hummel FC. Motor performance is not enhanced by daytime naps in older adults. Front Aging Neurosci. 2016; 8(MAY). https://doi.org/10.3389/ fnagi.2016.00125.

74. Debarnot U, Castellani E, Valenza G, Sebastiani L, Guillot A. Daytime naps improve motor imagery learning. Cogn Affect Behav Neurosci. 2011;11(4):541-50.

75. Debarnot U, Castellani E, Guillot A, Giannotti V, Dimarco M, Sebastiani L. Declarative interference affects off-line processing of motor imagery learning during both sleep and wakefulness. Neurobiol Learn Mem. 2012;98(4):361-7.

76. Brown RM, Robertson EM. Off-line processing: Reciprocal interactions between declarative and procedural memories. $\mathrm{J}$ Neurosci. 2007;27(39):10468-75.

77. Rothkirch I, Wolff S, Margraf NG, Pedersen A, Witt K. Does post-task declarative learning have an influence on early motor memory consolidation over day? An fMRI study. Front Neurosci. 2018; 12(APR). https://doi.org/10.3389/fnins.2018.00280.

78. Roveda E, et al. Effect of chronotype on motor skills specific to soccer in adolescent players. Chronobiol Int. 2020;37(4):552-63.

79. De Beukelaar TT, Van Soom J, Huber R, Wenderoth N. A day awake attenuates motor learning-induced increases in corticomotor excitability. Front Hum Neurosci. 2016;10(MAR2016):1-11.

80. Tucker MA, et al. The relative impact of sleep and circadian drive on motor skill acquisition and memory consolidation. Sleep. 2017; 40(4). https://doi.org/10.1093/sleep/zsx036.

81. Rulleau T, Mauvieux B, Toussaint L. Influence of circadian rhythms on the temporal features of motor imagery for older adult inpatients. Arch Phys Med Rehabil. 2015;96(7):1229-34.

82. Rulleau T, Robin N, Abou-Dest A, Chesnet D, Toussaint L. Does the improvement of position sense following motor imagery practice vary as a function of age and time of day? Exp Aging Res. 2018;44(5):443-54.

83. Vourvopoulos A, Niforatos E, Hlinka M, Skola F, Liarokapis F. Investigating the effect of user profile during training for BCIbased games, in 2017 9th International Conference on Virtual Worlds and Games for Serious Applications, VS-Games 2017 - Proceedings, 2017.

84. Fekih S, Zguira MS, Koubaa A, Masmoudi L, Bragazzi NL, Jarraya M. Effects of motor mental imagery training on tennis service performance during the ramadan fasting: a randomized, controlled trial, Nutrients. 2020, 12(4). https://doi.org/10.3390/ nu 12041035

85. Wittbrodt MT, Millard-Stafford M. Dehydration impairs cognitive performance: a meta-analysis. Med Sci Sports Exerc. 2018;50(11):2360-8. 
86. Meng $\mathrm{J}$, et al. Effects of soft drinks on resting state eeg and brain-computer interface performance. IEEE Access. 2017;5:18756-64.

87. Mang CS, Snow NJ, Wadden KP, Campbell KL, Boyd LA. Highintensity aerobic exercise enhances motor memory retrieval. Med Sci Sports Exerc. 2016;48(12):2477-86.

88. Marin Bosch B, et al. Effect of acute physical exercise on motor sequence memory. Sci Rep. 2020;10(1):1-13

89. Chapman CD, Benedict C, Schiöth HB. Experimenter gender and replicability in science. Sci Adv. 2018; 4(1). https://doi.org/ 10.1126/sciadv.1701427.

90.• Wood G, Kober SE. EEG neurofeedback is under strong control of psychosocial factors. Appl Psychophysiol Biofeedback. 2018;43(4):293-300. https://doi.org/10.1007/s10484-018-94073. Study effects of experimenter and participant gender in a large sample. Of all possible experimenter gender/participant gender combinations, only female participants with female experimenters were found to be not able to upregulate SMR.

91. Roc A, Pillette L, N'Kaoua B, Lotte F. Would motor-imagery based BCI user training benefit from more women experimenters? arXiv. 2019. https://doi.org/10.3217/978-3-85125-682-6-36.

92. Cardozo P, Cibeira LF, Rigo LC, Chiviacowsky S. Explicit and implicit activation of gender stereotypes additively impair soccer performance and learning in women. Eur J Sport Sci. 2020;0(0):1-8

93. Stone J, McWhinnie C. Evidence that blatant versus subtle stereotype threat cues impact performance through dual processes. J Exp Soc Psychol. 2008;44(2):445-52.

94. Schlatter $\mathrm{S}$, et al. Acute stress affects implicit but not explicit motor imagery: a pilot study. Int J Psychophysiol. 2020;152:62-71.

95. Dolfen N, King BR, Schwabe L, Swinnen S, Albouy G. Glucocorticoid response to stress induction prior to learning is negatively related to subsequent motor memory consolidation. Neurobiol Learn Mem. 2019;158(January):32-41.

96. Hordacre B, Immink MA, Ridding MC, Hillier S. Perceptualmotor learning benefits from increased stress and anxiety. Hum Mov Sci. 2016;49:36-46.

97. Kleih S. Motivation and SMR-BCI: fear of failure affects BCI performance, ... Fifth .... 2013; pp. 80-81.

98. Kleih SC, Riccio A, Mattia D, Kaiser V, Friedrich EVC, Scherer R. Motivation influences performance in SMR-BCI, G.R. Müller-Putz, R. Scherer, M. Billinger, A. Kreilinger, V. Kaiser, C. Neuper Proc. 5th Int. Brain-Computer Interface Conf., no. November 2014; 2011, pp. 108-111.

99. Nijboer F, et al. An auditory brain-computer interface (BCI). J Neurosci Methods. 2008;167(1):43-50.

100. Nijboer F, Birbaumer N, Kübler A. The influence of psychological state and motivation on brain-computer interface performance in patients with amyotrophic lateral sclerosis - a longitudinal study. Front Neurosci. 2010. https://doi.org/10.3389/ fnins.2010.00055.

101. Deci EL, Ryan RM. Self-determination theory: a macrotheory of human motivation, development, and health. Can Psychol. 2008;49(3):182-5.

102. Gonzalez DH, Chiviacowsky S. Relatedness support enhances motor learning. Psychol Res. 2018;82(3):439-47.

103. Grosse-Wentrup M, Schölkopf B. High gamma-power predicts performance in sensorimotor-rhythm brain-computer interfaces.
J Neural Eng. 2012; 9(4). https://doi.org/10.1088/1741-2560/9/ 4/046001.

104. Bamdadian A, Guan C, Ang KK, Xu J. The predictive role of pre-cue EEG rhythms on MI-based BCI classification performance. J Neurosci Methods. 2014;235:138-44.

105. Torkamani-Azar M, Jafarifarmand A, Cetin M. Prediction of motor imagery performance based on pre-trial spatio-spectral alertness features. Proc. Annu. Int. Conf. IEEE Eng. Med. Biol. Soc. EMBS; 2020; 2020-July, pp. 3062-3065.

106. Meinel A, Castaño-Candamil S, Reis J, Tangermann M. Pre-trial EEG-based single-trial motor performance prediction to enhance neuroergonomics for a hand force task. Front Hum Neurosci. 2016;10(APR2016):1-17.

107. Johnstone A, Grigoras I, Petitet P, Capitão LP, Stagg CJ. A single, clinically relevant dose of the GABAB agonist baclofen impairs visuomotor learning. J Physiol. 2020;0:1-16.

108. Ang KK, et al. Facilitating effects of transcranial direct current stimulation on motor imagery brain-computer interface with robotic feedback for stroke rehabilitation. Arch Phys Med Rehabil. 2015;96(3):S79-87.

109. Baxter BS, Edelman BJ, Sohrabpour A, He B. Anodal transcranial direct current stimulation increases bilateral directed brain connectivity during motor-imagery based brain-computer interface control. Front Neurosci. 2017;11(DEC):1-17.

110. Hong X, et al. Brain plasticity following MI-BCI training combined with tDCS in a randomized trial in chronic subcortical stroke subjects: a preliminary study. Sci Rep. 2017;7(1):1-12.

111. Chew E, et al. Using transcranial direct current stimulation to augment the effect of motor imagery-assisted brain-computer interface training in chronic stroke patients - cortical reorganization considerations. Front Neurol. 2020;11(August):1-11.

112. Naros G, Gharabaghi A. Physiological and behavioral effects of $\beta$-tACS on brain self-regulation in chronic stroke. Brain Stimul. 2017;10(2):251-9.

113. Halder $\mathrm{S}$, et al. Prediction of brain-computer interface aptitude from individual brain structure. Front Hum Neurosci. 2013. https://doi.org/10.3389/fnhum.2013.00105.

114. Meekes J, Debener S, Zich C, Bleichner MG, Kranczioch C. Does fractional anisotropy predict motor imagery neurofeedback performance in healthy older adults? Front Hum Neurosci. 2019;13(February):1-11.

115. Pascual-Leone A, Nguyet D, Cohen LG, Brasil-Neto JP, Cammarota A, Hallett M. Modulation of muscle responses evoked by transcranial magnetic stimulation during the acquisition of new fine motor skills. J Neurophysiol. 1995;74(3):1037-45.

116. Karni A, Tanne D, Rubenstein BS, Askenasy JJM, Sagi D. Dependence on REM sleep of overnight improvement of a perceptual skill. Science (80-.). 1994. https://doi.org/10.1126/scien ce.8036518.

117. Karni A, et al. The acquisition of skilled motor performance: fast and slow experience-driven changes in primary motor cortex. Proc Natl Acad Sci U S A. 1998. https://doi.org/10.1073/pnas. 95.3.861.

Publisher's Note Springer Nature remains neutral with regard to jurisdictional claims in published maps and institutional affiliations. 